\title{
The Ion Delivery Manner Influences the Antimicrobial Efficacy of Silver Oligodynamic lontophoresis
}

\author{
George Z. Tan ${ }^{1} \cdot$ Paul E. Orndorff ${ }^{2} \cdot$ Rohan A. Shirwaiker $^{3}$
}

Received: 25 April 2018 / Accepted: 7 September 2018 / Published online: 14 September 2018

(c) The Author(s) 2018

\begin{abstract}
Introduction Electrical activation of silver ions, known as oligodynamic iontophoresis, has shown broad-spectrum antimicrobial activities against bacteria, fungi, and viruses. However, it is not clear how the ion delivery manner, which is controlled by the electrical activation, influences the iontophoresis process. This paper focuses on this knowledge gap, aiming to characterize the interactive effects of electric current intensity and activation duration on the antimicrobial efficacy of a silver-based iontophoresis prototype against Gram-positive (S. aureus) and Gram-negative (E. coli) strains respectively.

Materials and Methods The modified Kirby-Bauer disc diffusion method was adopted to quantify the antimicrobial efficacy. A linear regression model was established and validated by empirical data.

Conclusion This study revealed that the antimicrobial activities of the device was more sensitive to current duration than current intensity, and the marginal antimicrobial efficacy of the device decreased as the current intensity increased. In addition, a sustained release of $\mathrm{Ag}+$ had superior antimicrobial efficacy compared to a fast release. These findings will contribute to the performance optimization of silver oligodynamic iontophoresis devices for antimicrobial applications.
\end{abstract}

Keywords Oligodynamic iontophoresis $\cdot$ Silver ion $\cdot$ Antimicrobial activities $\cdot$ Electrical activation

\section{Introduction}

Silver and its compounds have been used in medicine for decades as antimicrobial components of wound dressings, external skin treatments, debridement agents and eye medicines [1]. The use of silver in coatings currently spans from central venous catheters to urinary tract catheters and coated orthopaedic implants [2,3]. While the exact mechanism by which silver demonstrates its efficacy is still under investigation, it is widely believed that the antimicrobial effect is due to its ionic form [4]. In most existing silver-based

George Z. Tan

george.z.tan@ttu.edu

$\triangle$ Rohan A. Shirwaiker rashirwaiker@ncsu.edu

1 Department of Industrial, Manufacturing, and System Engineering, Texas Tech University, PO 43021, Lubbock, TX 79409-3061, USA

2 Department of Population Health and Pathobiology, North Carolina State University, Raleigh, NC, USA

3 Edward P. Fitts Department of Industrial and Systems Engineering, North Carolina State University, Campus Box 7906, 400 Daniels Hall, Raleigh, NC 27695-7906, USA antimicrobial products, the silver ions $\left(\mathrm{Ag}^{+}\right)$are passively diffused from the incorporated surface layer [5]. It has been found through in vivo animal models and clinical experiences that medical prostheses coated with silver alone do not necessarily demonstrate a discernible antimicrobial effect [6]. Thus the slow silver ion release rate becomes the major bottleneck of antimicrobial efficacy for most existing silver medical implants.

The oligodynamic effect was discovered in 1893 by the Swiss, Karl Wilhelm von Nägeli, as a toxic effect of heavy metal ions on living cells even in relatively low concentrations [7]. To accelerate the oligodynamic process, Spadaro et al. connected silver to the positive electrode (anode) of a power source and activated the release of $\mathrm{Ag}^{+}$by low intensity direct current (LIDC, $0.02-20 \mu \mathrm{A}$ ) [8]. This process is known as oligodynamic iontophoresis [9]. A series of in vitro experiments confirmed the high efficacy of this configuration in inhibiting bacterial growth. The inhibitory and antimicrobial concentrations of LIDC generated $\mathrm{Ag}^{+}$were 10-100 times lower than those for silver sulfadiazine. This finding led to explorations of silver medical devices based on LIDC activated silver ion action. The ion release process is essentially based on electrolysis, which is the passage of 
a direct electric current through metallic electrodes in a suitable solvent, resulting in ion exchange between the anode and cathode. According to Faraday's first law of electrolysis, by controlling the current intensity and duration, the concentration and the release rate of $\mathrm{Ag}^{+}$can be determined [10].

Electric current has been studied the most in similar LIDC activated silver-based systems since it is the driving force for release of the ions. Previous studies have shown a positive association between the current intensity and the antimicrobial efficacy of the system [11-14]. This finding is consistent with the Faraday's law that the amount of substance liberated at the anode is directly proportional to the electric charge passing through the system [15]. However, it is not clear whether the antimicrobial efficacy relies solely on the amount of $\mathrm{Ag}^{+}$regardless of the manner in which they are released. In an LIDC activated system, the electric charge is the product of the current intensity and the duration. Hence, the interactions of these two parameters are the effects of main interest in the study.

The objective of this study is to characterize the effects of electric current intensity and duration on the short-term antimicrobial efficacy of an LIDC activated silver-based prototype for biomedical application. The interactions of electric current intensity and duration were investigated using an agar test model, which was an adaptation of the standard Kirby-Bauer disc diffusion method for evaluating antibiotics [16].

\section{Materials and Methods}

\subsection{System Design}

There were four central components in the system: power source, silver anode, titanium cathode, and insulating electrode divider (Fig. 1). The silver was connected to the DC power source (batteries) as the anode to provide antimicrobial activities surrounding the device by releasing controlled amounts of $\mathrm{Ag}^{+}$. Despite the potential difference between

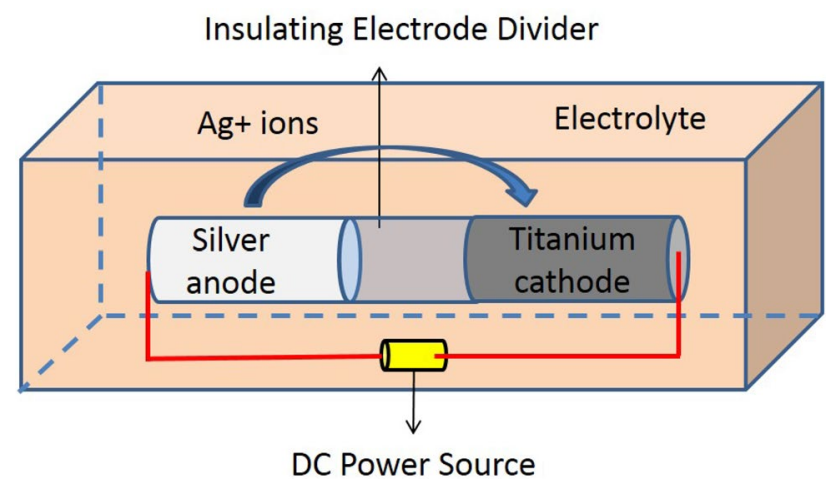

Fig. 1 Key components of a basic DIS the electrodes, the system was in a passive state (no system current) before it was placed in electrolyte due to the insulating divider between electrodes. After immersion, the electrolyte completed the circuit between the electrodes resulting in the active release of $\mathrm{Ag}^{+}$from the anode. Once the circuit was completed, the actual current intensity was measured by a multimeter.

\subsection{Antimicrobial Efficacy Test}

In this test model, the prototype was simplified to two separate metal wire electrodes connecting to an external DC source so that the testing was easy to operate. The electrodes were fully embedded within the Mueller-Hinton (MH) agar (Sigma-Aldrich, St. Louis, MO) plates $(100 \times 15 \mathrm{~mm})$ without direct contact, thus allowing the need for a separate insulating electrode divider in the test model. The top surface of agar was inoculated with bacteria. If the bacteria were sensitive to the ions released, an inhibition zone (IZ), seen as a clear ring, would form around the anode while the rest of the top surface area of the agar would be covered by bacterial colonies. The degree of antimicrobial efficacy could be interpreted from the IZ area.

Two small holes were punched on diametrically opposite locations on the side wall of the petri dish using a heated needle $(\varnothing 0.6 \mathrm{~mm})$, about halfway along the agar thickness. Silver and titanium electrode wires $(\varnothing 0.5 \mathrm{~mm}$,) were inserted into the agar along an orthogonal line and connected to the external breadboard using copper connecting wires. The resistors were inserted on the breadboard and connected with the $\mathrm{Ag}$ wire in series. The resistance of the $\mathrm{MH}$ agar was estimated to be $200 \mathrm{k} \Omega$. Through the breadboard, the silver wire was connected to the positive lead of the battery, and titanium wire to the negative lead. A detailed schematic of the setup was presented in Fig. 2a.

Escherichia coli (E. coli, MG 1655) and Staphylococcus aureus (S. aureus, ATCC 25213) were the two primary bacteria species tested in this stduy. Isolated bacteria colonies were grown overnight at $37^{\circ} \mathrm{C}$ from refrigerated samples on an $\mathrm{MH}$ agar plate. The desired starting inoculant concentration values for the agar test was $10^{7} \mathrm{CFU} / \mathrm{ml}$. The inoculant was prepared in $0.7 \%$ phosphate buffered saline (PBS). The original bacterial concentration was estimated by the spectrophotometer (Shimadzu Inc, Norcross, GA; $\lambda=600 \mathrm{~nm}$ ) to be $1-5 \times 10^{8} \mathrm{CFU} / \mathrm{ml}$, and then diluted by one tenth.

$100 \mu \mathrm{l}$ of the bacteria-inoculated PBS was pipetted onto the surface of agar and smeared uniformly over the surface in 3 directions using a cotton swab. By weighing the used swabs before and after agar inoculation, the average volume of the PBS on agar was estimated to be $50 \mu$ l. The electrical circuit was turned on to activate the DIS, and the entire setup (Fig. 2b) was incubated at $37^{\circ} \mathrm{C}$ for the specified test interval and without electricity for overnight thereafter. The 
Fig. 2 a A schematic of the agar test configuration, $\mathbf{b}$ an actual experimental setup of the agar test (a)

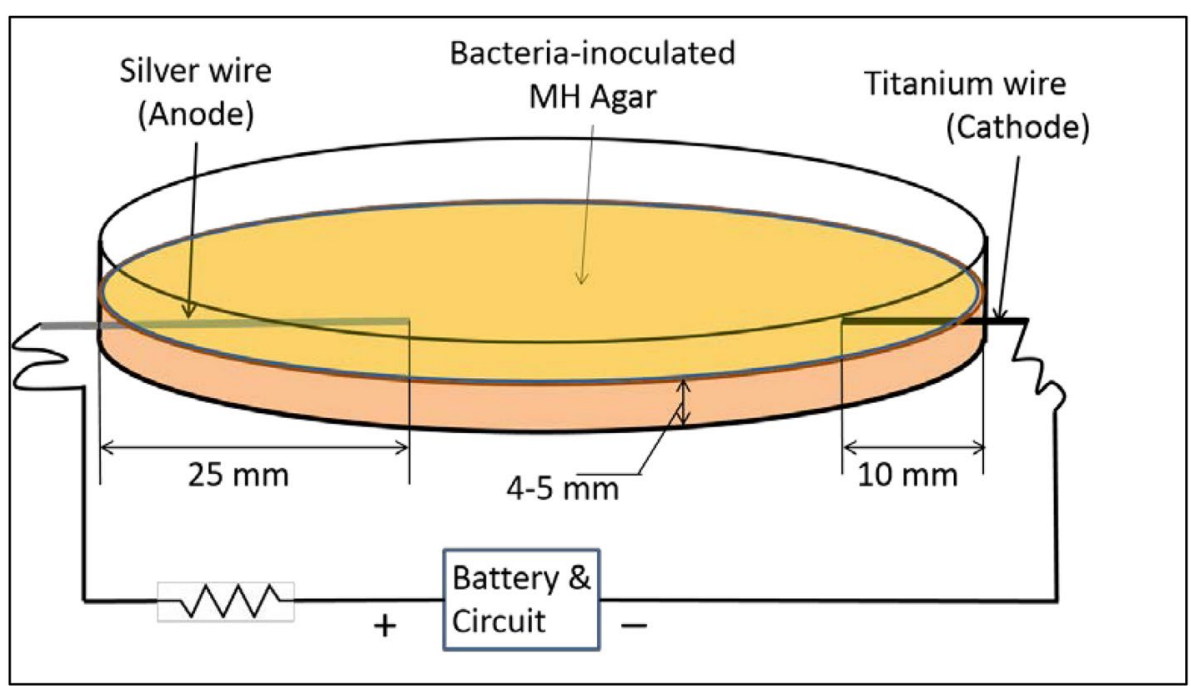

(b)

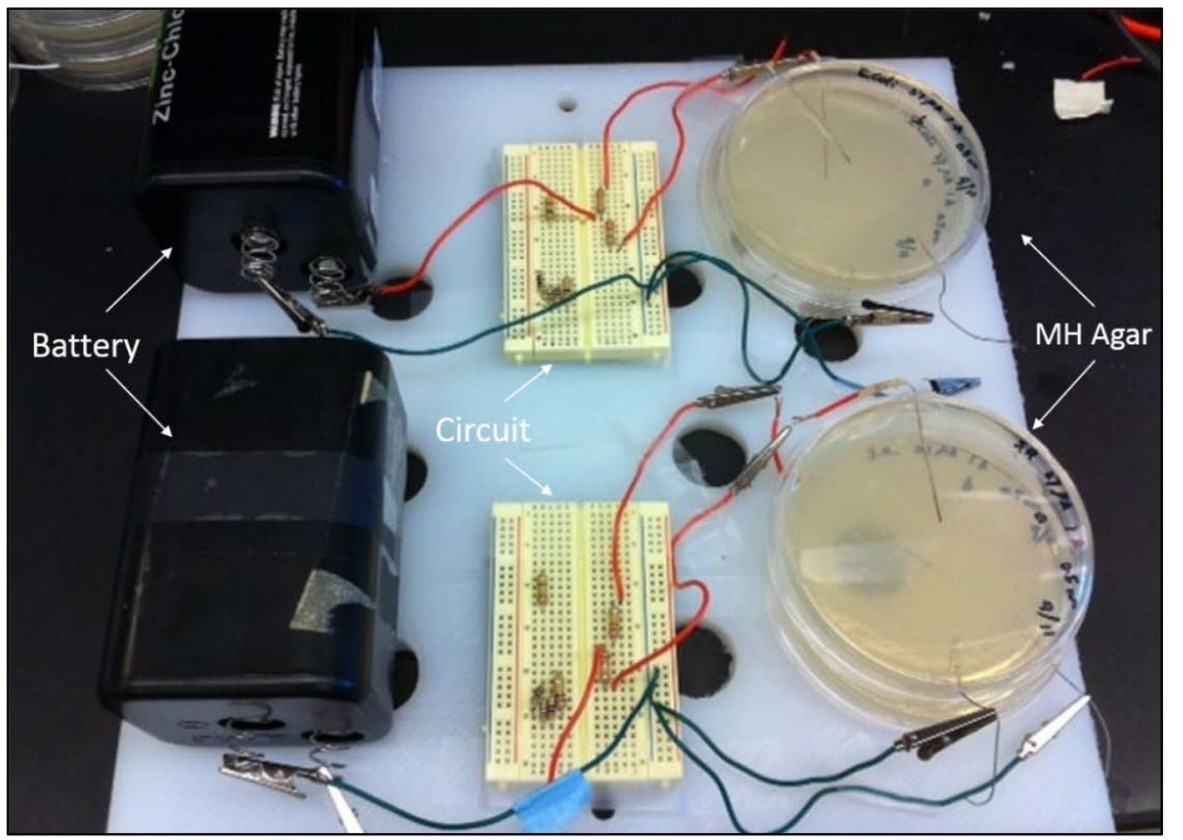

area of the IZ was estimated by multiplying its averaged width by length.

\subsection{Design of Experiments}

A randomized block experimental design was adopted in which bacteria type (E. coli and S. aureus) served as the blocking factor (a source of variability that is not of primary interest). Three levels were tested for each main factor $-1.2 \mu \mathrm{A}, 5 \mu \mathrm{A}$ and $20 \mu \mathrm{A}$ for current intensity, and $0.25 \mathrm{~h}, 1 \mathrm{~h}$ and $4 \mathrm{~h}$ for duration. The purpose of adopting such low levels was to determine the lower bound of the parameter settings to achieve a significant antimicrobial efficacy. The current intensity was determined by proper combination of the resistors (Table 1). The differences between theoretical values and actual values were due to the errors of voltage and resistance within the specification tolerances. All the differences were below 3.5\%, and thus negligible. Therefore it is appropriately to use the theoretical values for modeling. Three replicates were used for each of the nine combinations in every block resulting in a total of 54 agar plate-based antimicrobial efficacy experiments.

A regression model was built based on the empirical data and a set of validation experiments was carried out to evaluate the reliability of the regression model for the antimicrobial efficacy. The levels of the factors were set to be at the quarter points of the original experimental design. There were two samples in each group. 
Table 1 Levels of current intensity in 2D agar test model

\begin{tabular}{lllc}
\hline $\begin{array}{l}\text { Set values of cur- } \\
\text { rent intensity }(\mu \mathrm{A})\end{array}$ & Actual value of battery voltage & Resistor combinations & $\begin{array}{l}\text { Actual values of } \\
\text { current intensity } \\
(\mu \mathrm{A})\end{array}$ \\
\hline 1.2 & $6.2 \mathrm{~V}$ & Two $10 \mathrm{M} \Omega$ resistors in parallel & 1.19 \\
5 & & One $1 \mathrm{M} \Omega$ resistor & 5.16 \\
20 & & One $1 \mathrm{k} \Omega$ resistor & 20.67 \\
\hline
\end{tabular}

\subsection{Statistical Models}

The effects of the investigated factors, current intensity and duration, were modeled using a two-way ANOVA model with the response variable $y$ representing the IZ area, $B$ representing the blocking effect, $\alpha$ and $\beta$ representing the effects of current intensity and current duration respectively. Statistical significance $(p<0.05)$ was determined using the PROC GLM Procedure (SAS 9.2 for Windows).

A regression model was established to estimate the relationships among variables using the maximum improvement $\mathrm{R}^{2}$ (MAXR) method [17]. The blocking factor (bacterial species) was transferred into a numeric form with $E$. coli $=1$ and $S$. aureus $=2$. The regression model was first established by fitting current intensity and duration with original values $(1.2,5,20$ and $0.25,1,4)$.

$y_{i j k}=\mu+B_{i}+\alpha_{j}+\beta_{k}+\alpha \beta_{j k}+\varepsilon_{i j k}$

where $i=1,2$ (index for blocks), $j=1,2,3$ (index for current intensity), $k=1,2,3$ (index for current duration), $\varepsilon_{i j k} \sim \operatorname{iid} N\left(0, \sigma^{2}\right)$.

A second model was then built with these two variables in common logarithmic form $(0.08,0.7,1.3$ and -0.6, 0, 0.6). The analysis was conducted using the PROC REG Procedure (SAS 9.2 for Windows).

\section{Results}

Sample images of agar plates from the experiments on $E$. coli and $S$. aureus are presented in Fig. 3a, b respectively. The rounded rectangle shape of IZ indicates that the diffusion of $\mathrm{Ag}^{+}$in the agar is almost uniform along the length of the silver anode.

Figure 4 gives the histogram of the IZ area. The positive effects of current intensity and duration on the antimicrobial efficacy can be observed from the graph. The lower bounds of duration to achieve remarkable antimicrobial efficacy $\left(\mathrm{IZ}>100 \mathrm{~mm}^{2}\right.$ ) at $1.2 \mu \mathrm{A}, 5 \mu \mathrm{A}$ and $20 \mu \mathrm{A}$ are $4 \mathrm{~h}, 1 \mathrm{~h}$ and $0.25 \mathrm{~h}$, respectively. The mean values of IZ area as well as the standard deviations are summarized in Table 2. E. coli was more sensitive to the DIS than S. aureus. For $E$. coli, treatment of the DIS with $1.2 \mu \mathrm{A}$ for $0.25 \mathrm{~h}$ leads to a discernible IZ $\left(151.7 \mathrm{~mm}^{2}\right)$. However, for $S$. aureus, treatment at this level can hardly result in a detectable IZ.

The ANOVA results are summarized in Table 3. The goodness-of-fit of the statistical model is validated by a high $\mathrm{R}^{2}$ of 0.8506 . The $p$-values of all factors, including the interaction effect, are less than 0.05 . The antimicrobial efficacy of the system (IZ area) was significantly influenced by the bacterial specie, the current intensity, the current duration and the interaction effect between intensity and duration.

Table 4 summarizes the results of regression analysis. The model with logarithmic variables possesses better goodness-of-fit $\left(\mathrm{R}^{2}=0.8411\right)$ compared to the one with original variables $\left(R^{2}=0.7840\right)$. The regression model for $2 \mathrm{D}$ agar test is given as follows:

$$
\begin{aligned}
I Z= & 355.66-128.24 \cdot B S+58.25 \cdot \log _{10}(D) \\
& +12.71 \cdot \log _{10}(I)-12.23 \cdot \log _{10}(D) \log _{10}(I)
\end{aligned}
$$

where $B S=$ bacterial species, $D=$ current duration, $I=$ current intensity

In Fig. 5, the surface approximation graph visualizes the unbalanced positive influences of duration and intensity. In addition, the logarithmic relationship is an implication of the diminishing marginal effectiveness of these two factors. The results of the validation experiments are summarized in Table 5. It is observed that the theoretical values for all groups show an accuracy of over $80 \%$. The regression model is particularly reliable (error $=0.01$ ) for describing the antimicrobial efficacy of the silver oligodynamic iontophoresis against $S$. aureus.

\section{Discussion}

Bone cement loaded with metallic silver nanoparticles has shown good antimicrobial activity against Methicillin-resistant Staphylococcus epidermidis (MRSE) and MRSA, which Gentamicin cement was not effective against due to the highlevel gentamicin resistance [18]. Silver-loaded coatings on titanium surface, titanium oxide surface and hydroxyapatite (HAp) film have demonstrated antimicrobial performance against $S$. aureus, S. epidermidis, E. coli and Pseudomonas aeruginosa, while retained good biocompatibility [19-24]. However, Fordham et al. evaluated the in vitro bactericidal 
Fig. 3 Sample agar plates from experiments on $\mathbf{a} E$. coli, and $\mathbf{b}$ S. aureus (a)

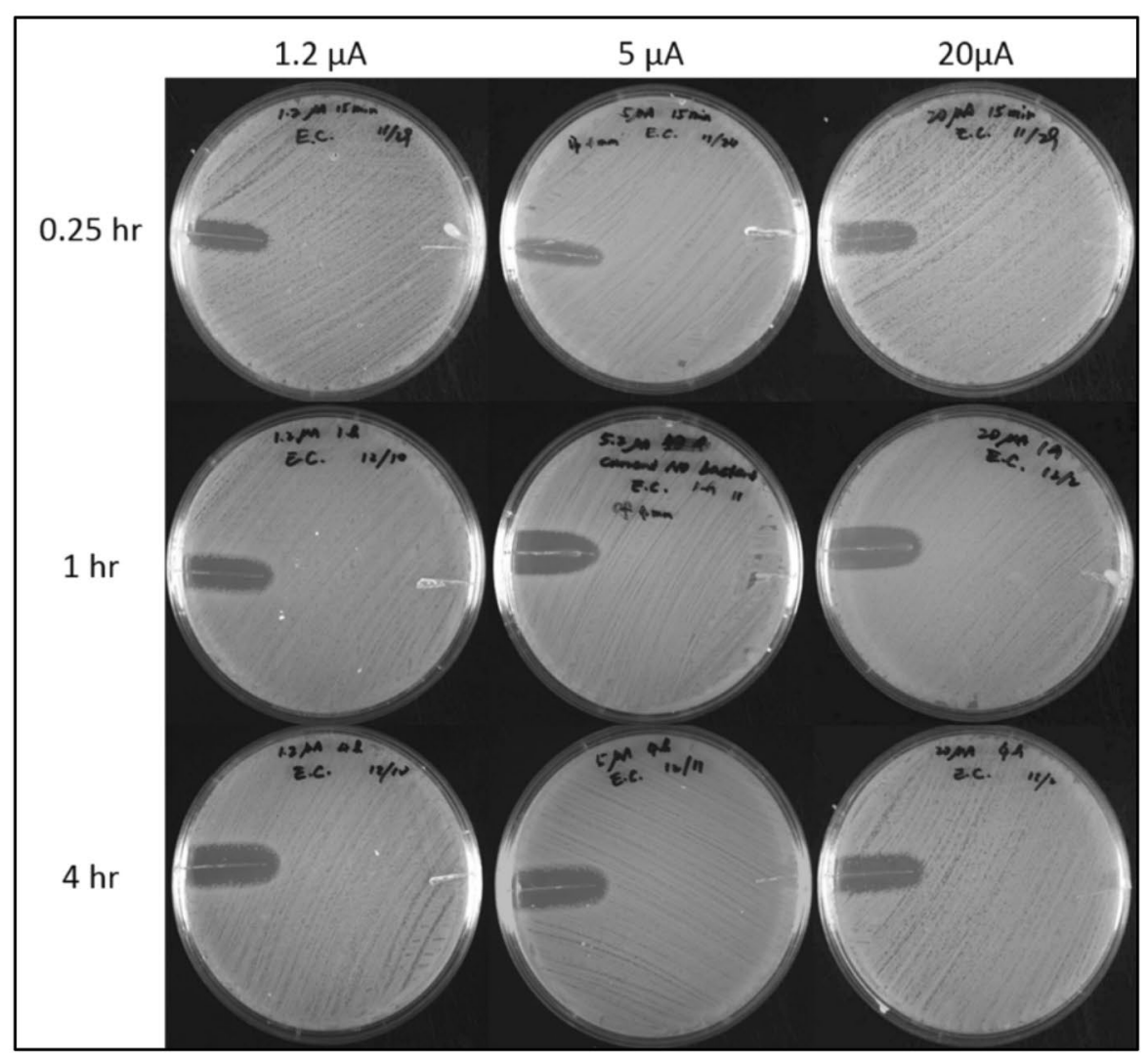

(b)

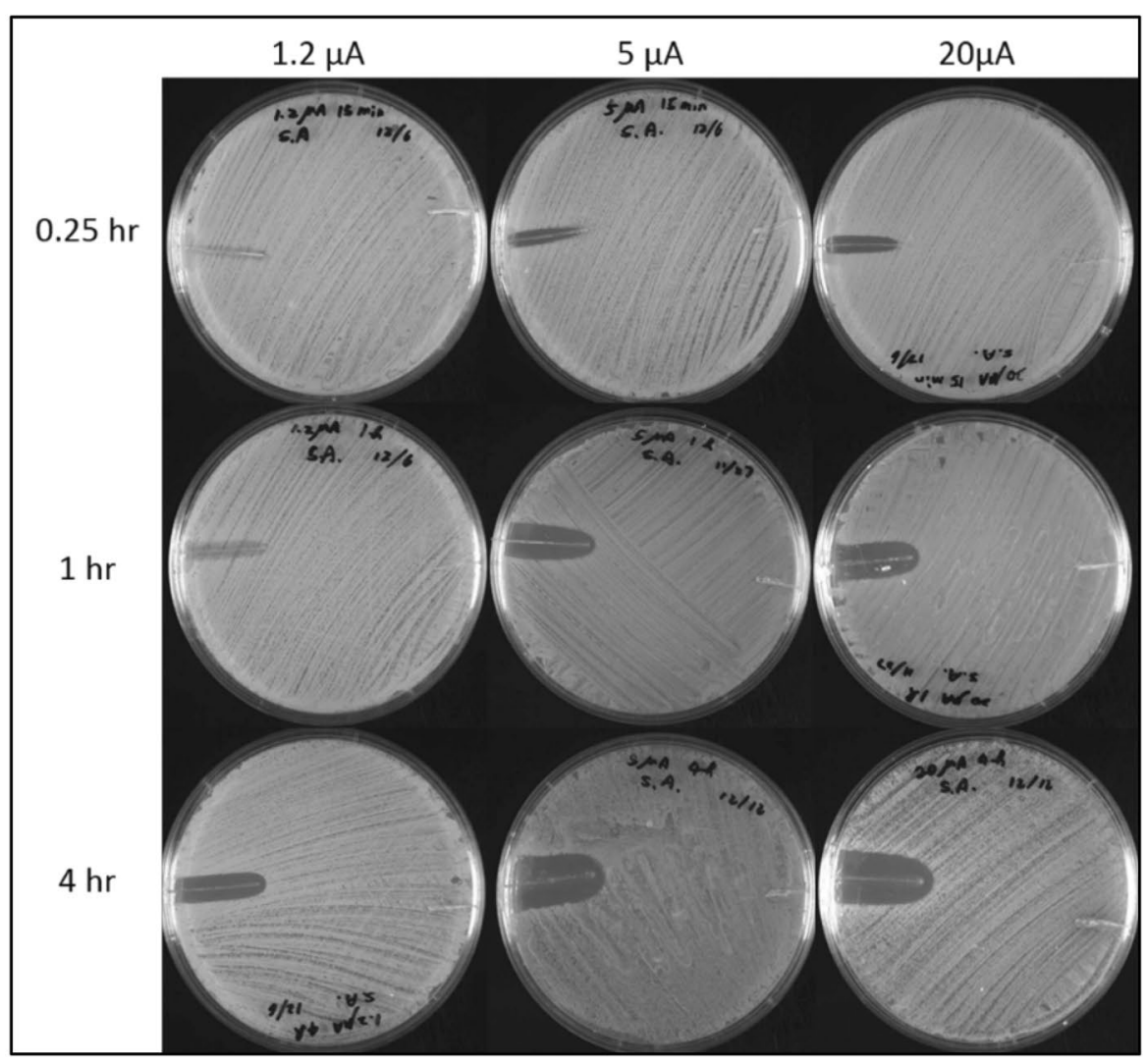


Fig. 4 Histogram of IZ areas in 2D agar study

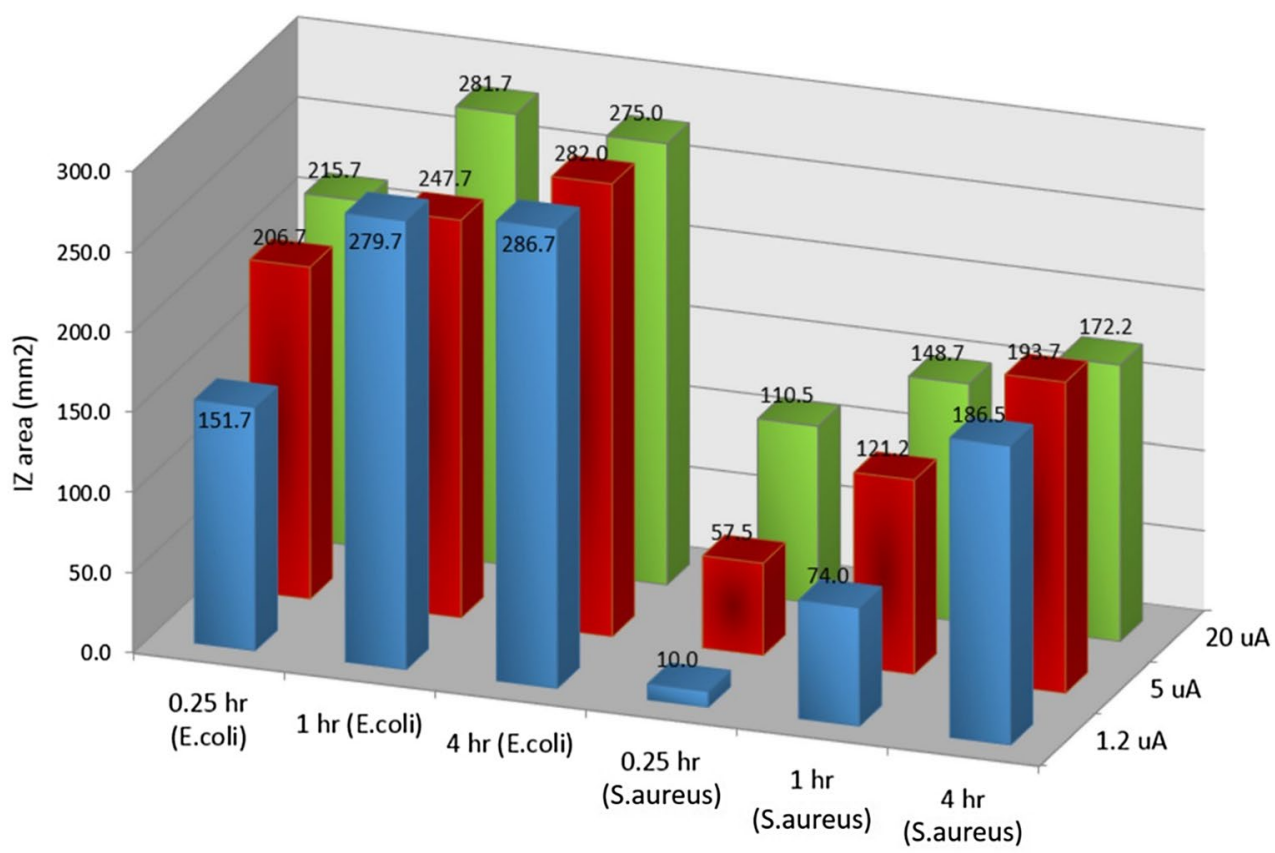

Table 2 Mean values and standard deviation of IZ area in 2D agar study

\begin{tabular}{llllc}
\hline Factors & Levels & \# of samples & IZ Mean $\left(\mathrm{mm}^{2}\right)$ & $\begin{array}{l}\text { IZ Std } \\
\text { Dev } \\
\left(\mathrm{mm}^{2}\right)\end{array}$ \\
\hline Bacteria & E. coli & 27 & 247.4 & 53.8 \\
& S. aureus & 27 & 119.4 & 67.6 \\
Intensity $(\mu \mathrm{A})$ & 1.2 & 18 & 164.8 & 111.0 \\
& 5 & 18 & 184.8 & 80.6 \\
& 20 & 18 & 200.6 & 70.3 \\
Duration $(\mathrm{h})$ & 0.25 & 18 & 125.3 & 79.1 \\
& 1 & 18 & 192.1 & 92.4 \\
& 4 & 18 & 232.7 & 58.7 \\
\hline
\end{tabular}

performance of silver coating for biomedical implants and suggested that pure silver is an inadequate bactericide for orthopedic implants inserted in the medullary canal [25]. The antimicrobial efficacy of medical devices with passive silver thin films is still under discussion.

The absence of control in the ion diffusion mechanism impedes further development of safe local administration of antimicrobial silver ions. On one side, products with low silver concentration may not demonstrate significant local antimicrobial efficacy. On the other, long exposure periods of implants with high silver concentration will increase the risk of system hazard and induce silver-resistance species. A possible solution to this dilemma is the application of
Table 3 ANOVA results of antimicrobial test

\begin{tabular}{llllll}
\hline Dependent & Variable & IZ & & & \\
\hline Source & DF & Squares & Mean square & F value & Pr $>$ F \\
Model & 9 & 353529.13 & 39281.01 & 27.84 & $<.0001$ \\
Error & 44 & 62071.33 & 1410.71 & & \\
Corrected total & 53 & 415600.47 & & & \\
$\mathrm{R}^{2}$ & Coeff var & Root MSE & IZ mean & & \\
0.850647 & 20.4818 & 37.56 & 183.38 & & \\
Source & DF & Type I SS & Mean square & F value & Pr $>$ F \\
Bacteria & 1 & 221376.04 & 221376.04 & 156.93 & $<.0001$ \\
Intensity & 2 & 11626.95 & 5813.48 & 4.12 & 0.0229 \\
Duration & 2 & 105755.56 & 52877.78 & 37.48 & $<.0001$ \\
Intensity $\times$ Duration & 4 & 14770.57 & 3692.64 & 2.62 & 0.0477 \\
\hline
\end{tabular}


Table 4 Results of regression model selection for $2 \mathrm{D}$ agar test

\begin{tabular}{lccccc}
\hline Variable & Parameter estimate & Standard error & Type II SS & F value & Pr $>$ F \\
\hline Original model: & $\mathrm{R}^{2}=0.7840$ and $\mathrm{C}(\mathrm{p})=5.000$ & & & \\
Intercept & 301.83 & 21.58 & 359733 & 195.53 & $<.0001$ \\
Bacteria & -128.24 & 11.67 & 222017 & 120.68 & $<.0001$ \\
Duration & 34.06 & 5.29 & 76201 & 41.42 & $<.0001$ \\
Current & 3.62 & 1.06 & 21523 & 11.7 & 0.0013 \\
Interact & -1.12 & 0.44 & 11699 & 6.36 & 0.015 \\
Log model: $\mathrm{R}^{2}=0.8411$ and C(p)=5.0000 & & & & \\
Intercept & 355.66 & 17.29 & 572537 & 423.22 & $<.0001$ \\
Bacteria & -128.24 & 10.01 & 222017 & 164.11 & $<.0001$ \\
LogD & 58.26 & 7.57 & 80128 & 59.23 & $<.0001$ \\
LogI & 12.71 & 4.36 & 11514 & 8.51 & 0.0053 \\
LogInter & -12.23 & 3.85 & 13662 & 10.1 & 0.0026 \\
\hline
\end{tabular}

Fig. 5 Surface approximation graph for the agar test

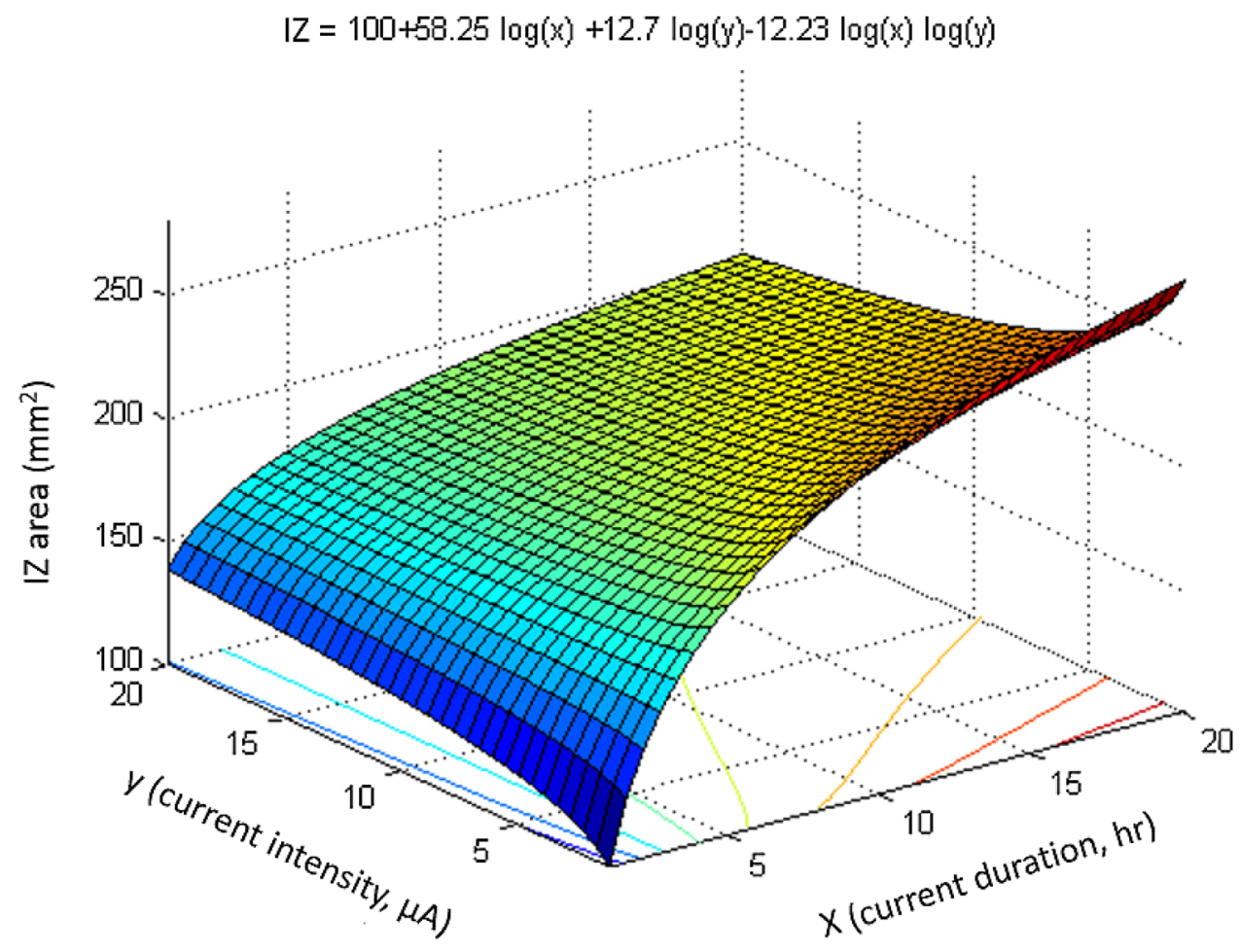

Table 5 Results of verification experiments for the regression model

\begin{tabular}{llllc}
\hline Bacteria & $\begin{array}{l}\text { Intensity }(\mu \mathrm{A}) \\
\text { and duration } \\
(\mathrm{h})\end{array}$ & $\begin{array}{l}\text { Theoretical IZ } \\
\text { means }\left(\mathrm{mm}^{2}\right)\end{array}$ & $\begin{array}{l}\text { Empirical IZ } \\
\text { means }\left(\mathrm{mm}^{2}\right)\end{array}$ & $\%$ Error \\
\hline E. coli & $2.5,0.5$ & 279.75 & 237 & 18 \\
E. coli & 10,2 & 350.84 & 293.25 & 20 \\
S. aureus & $2.5,0.5$ & 151.51 & 150 & 1 \\
S. aureus & 10,2 & 222.6 & 220 & 1 \\
\hline
\end{tabular}

oligodynamic iontophoresis, an active control mechanism which utilizes direct electric current to regulate the ion release.

The first human clinical study using electrically activated silver devices as a treatment for orthopaedic infection was reported by Becker et al. in 1978 [26]. In this six-week study, a pure silver wire was inserted directly into infected nonunions in which the wound was to be surgically close. The $8 \mathrm{~cm}$ long electrodes were insulated with Teflon except for the terminals. The electrode was first made DC positive with a current of $1 \mu \mathrm{A}$ for twenty-four hours after surgery. Then the current was reduced to $0.1 \mu \mathrm{A}$ and the electrode polarity was reversed without interruption until $1.5-2.5 \mathrm{~J}$ of total 
energy had been delivered. The treatment resulted in control of the infection in 12 of the 15 patients after follow-up ranging from 3 to 36 months. In the other three cases which failed to eradicate the infection, two remarkably reduced bacterial flora in the wound. No detectable toxic effect on local tissues was reported. In fact, deposition of substantial amounts of new bone was reported with the silver-Teflon anodes.

In a later clinical study conducted by Webster et al. 25 patients with active, chronic osteomyelitis, resistant to conventional management were treated with surgical debridement and daily application of electrically activated silver nylon dressings [27]. The dressing was placed in close contact with all exposed bone and soft tissue with the exception of tendons and ligaments. The tail portion of the silver nylon was connected to the positive terminal of a DC source unit. A skin pad (negative terminal) was taped firmly directly opposite to the wound. During the actual treatment, the driving voltage of the DC source was $0.8-0.6 \mathrm{~V}$ with a corresponding current between $100-250 \mu \mathrm{A}$ throughout the treatment period. This corresponded to a nominal current density of about $0.7-1.6 \mu \mathrm{A} / \mathrm{cm}^{2}$ in the actual silver nylon dressing. After 4 weeks of treatment, 16 cases $(64 \%)$ resulted in closed, stable, pain-free wounds. No side effects of anodic silver administration, toxic or otherwise, were noted in this study.

So far there is no previous study investigating the effect of ion delivery manner on the antimicrobial efficacy of the electrically-activated silver system. It is widely believed that the antimicrobial efficacy of the silver oligodynamic iontophoresis largely depends on the quantity of $\mathrm{Ag}^{+}$released from the anode, which is proportional to the total quantity of electric charge $\left(\mathrm{Q}_{\mathrm{E}}\right)$ transferred at the anode. However, the empirical results of the 2D agar study reveal an unbalanced interaction effect of the current intensity and the duration on the size of the IZ area, which indicates that the antimicrobial efficacy of the DIS is not only dependent on the quantity of the delivered $\mathrm{Ag}^{+}$, but also the manner of delivering. According to Faraday's laws of electrolysis, an electric activation of $1.2 \mu \mathrm{A}$ by $4 \mathrm{~h}(4.8 \mu \mathrm{A} \cdot \mathrm{h})$ generates marginally less $\mathrm{Ag}^{+}$than an activation of $5 \mu \mathrm{A}$ by $1 \mathrm{~h}(5 \mu \mathrm{A} \cdot \mathrm{h})$. However, in our study, for both bacterial strains, $1.2 \mu \mathrm{A}$ by $4 \mathrm{~h}$ resulted in larger IZs than $5 \mu \mathrm{A}$ by $1 \mathrm{~h}$. The same phenomenon was observed in all other comparisons. Generally, with the same level of total quantity of electricity $(\mu \mathrm{A} \cdot \mathrm{hr})$, low current intensity with long duration exhibited a higher antimicrobial efficacy than high current intensity with short duration. As such, this indicated that a sustained release of $\mathrm{Ag}^{+}$has superior antimicrobial efficacy compared to a fast release.

With certain parameters, the silver oligodynamic iontophoresis demonstrated different antimicrobial efficacies against gram-negative and gram-positive strains, respectively. For $E$. coli, the combination of $1 \mathrm{~h}$ and $1.2 \mu \mathrm{A}$ led to a larger IZ than that of $0.25 \mathrm{~h}$ and $20 \mu \mathrm{A}$ (279.7>215.7 from Fig. 4), however, for S. aureus, the results were opposite $(74<110.5)$. Theoretically, the combination of $1 \mathrm{~h}$ and $1.2 \mu \mathrm{A}(1.2 \mu \mathrm{A} \cdot \mathrm{h})$ released less total amount of $\mathrm{Ag}^{+}$compared to that of $0.25 \mathrm{~h}$ and $20 \mu \mathrm{A}$ $(5 \mu \mathrm{A} \cdot \mathrm{h})$. The discrepancy of $\mathrm{Ag}^{+}$effectiveness in this comparison indicates that under certain conditions, $S$. aureus is more sensitive to the total amount of released $\mathrm{Ag}^{+}$while the E.coli is more sensitive to the duration. Because the minimum inhibition concentration (MIC) of silver for E.coli is lower than that for S. aureus [28, 29], we hypothesize that before reaching the MIC, the antimicrobial efficacy (indicated by the IZ area) is positively correlated to the total amount of released $\mathrm{Ag}^{+}$, while after the MIC is achieved, the antimicrobial efficacy depends on the iontophoresis duration. This hypothesis needs to be tested by further mechanism-based studies.

In addition, we observed that for E.coli, with a duration of $1 \mathrm{~h}$ or $4 \mathrm{~h}$, an increase in current intensity did not necessarily result in an enhancement in antimicrobial efficacy. A similar phenomenon was observed in the 4-h test for S. aureus. These results indicate that there was an upper bound of IZ due to the limited capability of $\mathrm{Ag}^{+}$diffusion in the agar. Once the antimicrobial capability is saturated, increasing the current intensity or the duration will not substantially enlarge the IZ.

Another interesting phenomenon is that the shape of the IZ exhibited slightly different patterns. For most of the IZs, a rectangle shape was formed along the silver wire body, followed by a cone-shape tip at the pinpoint of the wire. When the duration increased to $4 \mathrm{~h}$, the cone-shaped tip tended to be more rounded. The shape of the IZ is determined by the silver concentration gradient. We can assume that the $\mathrm{Ag}^{+}$diffusion is negatively correlated to the distance, and each point at the silver electrode wire has the same $\mathrm{Ag}^{+}$diffusion rate with a given set of process parameters. As shown in Fig. 6, for a given point $(\mathrm{P})$ around the silver electrode wire, the silver concentration at $\mathrm{P}$ can be estimated as the integral of a decreasing function of the distances between $\mathrm{P}$ and all the points $(\mathrm{x})$ on the wire, namely Con. $(P)=\int_{0}^{b} f\left(\left(a^{2}+x^{2}\right)^{-1 / 2}\right) d x+\int_{0}^{L-b} f\left(\left(a^{2}+x^{2}\right)^{-1 / 2}\right) d x$. Apparently the silver concentration around the pinpoint of the wire will be lower than those around the wire body. When the duration is long enough, $\mathrm{Ag}^{+}$diffusion around the wire body reaches its maximum distance while the diffusion at the wire pinpoint continues to expand, leading to a rounded IZ tip.

This paper revealed that the antimicrobial efficacy of the system is more sensitive to the current duration than the intensity. It indicated that the sustained release of $\mathrm{Ag}^{+}$ has superior antimicrobial efficacy compared to the fast 

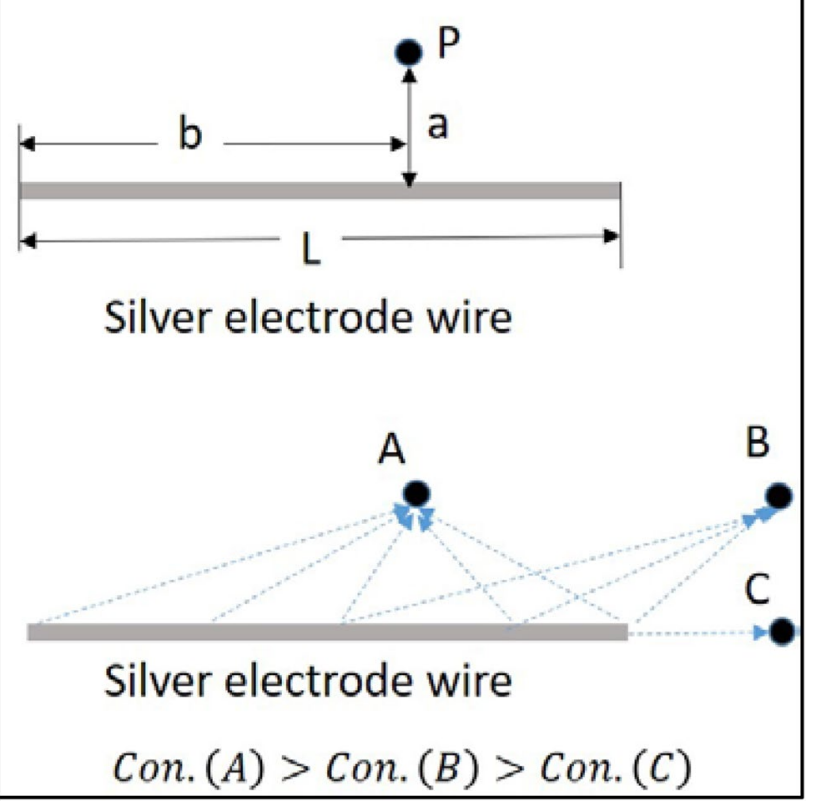

Fig. 6 An illustration of silver concentration around the anode wire

release. With the same $\mathrm{Q}_{\mathrm{E}}$, long-term current activation at lower intensity may result in better antimicrobial efficacy than short-term current activation at higher intensity. The logarithmic linear relation shows that the marginal effects of both factors decreases, further suggesting the lack of necessity of applying high current intensity in the DIS. It also implied that the incremental antimicrobial efficacy of the system decreases as the current intensity incrementally increases while all other factors remains constant.

It should be noted that the agar diffusion-based method is a semi-quantitative analysis. The main limitation of this study is that the antimicrobial efficacy was evaluated solely based on the area of the IZ irrespective of its clearness. For example. the combination of $1 \mathrm{~h}$ and $1.2 \mu \mathrm{A}$ had an IZ less clear than that from the combination of $5 \mu \mathrm{A}$ and $1 \mathrm{~h}$, indicating a remaining coverage of $S$. aureus, even though it had a larger IZ area $(74>57.5)$. In future, quantitative methods to study bacteria inhibition should be developed and utilized in future revision of the models.

\section{Conclusions}

This study characterized the effects of electric activation duration and current intensity on the short-term antimicrobial efficacy of a silver oligodynamic iontophoresis device using a modified Kirby-Bauer agar test model. It was found that the antimicrobial performance of the device was more sensitive to current duration than current intensity, and the marginal antimicrobial efficacy of the device decreased as the current intensity increased. Therefore it is recommended to utilize low-intensity and long-duration activation to achieve the optimal performance of the silver oligodynamic iontophoresis device for antimicrobial applications.

Open Access This article is distributed under the terms of the Creative Commons Attribution 4.0 International License (http://creativeco mmons.org/licenses/by/4.0/), which permits unrestricted use, distribution, and reproduction in any medium, provided you give appropriate credit to the original author(s) and the source, provide a link to the Creative Commons license, and indicate if changes were made.

\section{References}

1. Pugliese, G., \& Favero, M. S. (1999). Efficacy of silver-coating central venous catheters. Infection Control and Hospital Epidemiology, 20(6), 416.

2. Pollini, M., et al. (2011). Antibacterial coatings on haemodialysis catheters by photochemical deposition of silver nanoparticles. Journal of Materials Science Materials in Medicine, 22(9), 2005-2012.

3. Odekerken, J.C.E., et al. (2013). Modern orthopaedic implant coatings - their pro's, con's and evaluation methods, in Modern surface engineering treatments. InTech.

4. Ip, M., et al. (2006). Antimicrobial activities of silver dressings: An in vitro comparison. Journal of Medical Microbiology, 55(1), 59-63.

5. Castellano, J. J., et al. (2007). Comparative evaluation of silvercontaining antimicrobial dressings and drugs. International Wound Journal, 4(2), 114-122.

6. Darouiche, R. O. (1999). Anti-Infective efficacy of silvercoated medical prostheses. Clinical Infectious Diseases, 29(6), 1371-1377.

7. Rentz, E. J. (2003). Viral pathogens and severe acute respiratory syndrome: oligodynamic $\mathrm{Ag}+$ for direct immune intervention. Journal of Nutritional \& Environmental Medicine, 13(2), 109.

8. Spadaro, J. A., et al. (1974). Antibacterial effects of silver electrodes with weak direct current. Antimicrobial Agents and Chemotherapy, 6(5), 637-642.

9. Davis, C. P., et al. (1991). Bacterial and fungal killing by iontophoresis with long-lived electrodes. Antimicrobial Agents and Chemotherapy, 35(10), 2131-2134.

10. Fuller, T. A., et al. (2010). Developing an engineered antimicrobial/prophylactic system using electrically activated bactericidal metals. Journal of Materials Science Materials in Medicine, 21(7), 2103-2114.

11. Shirwaiker, R. A., et al. (2013). Nanomaterials and synergistic low-intensity direct current (LIDC) stimulation technology for orthopedic implantable medical devices. WIREs Nanomedicine and Nanobiotechnology, 5, 191-204.

12. Tan, Z., et al. (2016). Effects of electrically activated silvertitanium implant system design parameters on time-kill curves against Staphylococcus aureus. Journal of Medical and Biological Engineering, 36(3), 325-333.

13. Tan, Z., Orndorff, P. E., \& Shirwaiker, R. A. (2015). Modified Pharmacokinetic/Pharmacodynamic model for electrically activated silver-titanium implant system. Biomaterials and Biomechanics in Bioengineering, 2(3), 127-141.

14. Tan, Z., et al. (2017). Antibacterial efficacy and cytotoxicity of low intensity direct current activated silver-titanium implant system prototype. BioMetals, 30(1), 113-125. 
15. Serway, R.A., C.J. Moses, and C.A. Moyer (2004) Chapter 4: The particle nature of matter, in Modern physics. David Harris.

16. Biemer, J. J. (1972). Antimicrobial susceptibility testing by the Kirby-Bauer disc diffusion method. Annals of clinical laboratory science, 3(2), 135-140.

17. Procedures. (2009). In SAS/STAT(R) 9.2 user's guide. 2nd edn. SAS: Cary.

18. Alt, V., et al. (2004). An in vitro assessment of the antibacterial properties and cytotoxicity of nanoparticulate silver bone cement. Biomaterials, 25(18), 4383-4391.

19. Qin, H., et al. (2014). Anti-biofilm effects of silver nanoparticles immobilized on titanium. Biomaterials, 35(33), 9114-9125.

20. Tian, Y., et al. (2014). Antibacterial activity and cytocompatibility of titanium oxide coating modified by iron ion implantation. Acta Biomaterialia, 10(10), 4505-4517.

21. Kakinuma, H., et al. (2010). Antibacterial polyetheretherketone implants immobilized with silver ions based on chelate-bonding ability of inositol phosphate: Processing, material characterization, cytotoxicity, and antibacterial properties. Journal of Biomedical Materials Research Part A, 103(1), 57-64.

22. Cheng, H., et al. (2014). Long-lasting in vivo and in vitro antibacterial ability of nanostructured titania coating incorporated with silver nanoparticles. Journal of Biomedical Materials Research, Part A, 102(10), 3488-3499.

23. Uhm, S. H., et al. (2014). Fabrication of bioactive, antibacterial $\mathrm{TiO}_{2}$ nanotube surfaces, coated with magnetron sputtered $\mathrm{Ag}$ nanostructures for dental applications. Journal of Nanoscience and Nanotechnology, 14(10), 7847-7854.

24. Gosheger, G., et al. (2004). Silver-coated megaendoprostheses in a rabbit model: An analysis of the infection rate and toxicological side effects. Biomaterials, 25(24), 5547-5556.

25. Fordham, W. R., et al. (2014). Silver as a bactericidal coating for biomedical implants. Surface \& Coatings Technology, 253, 52-57.

26. Becker, R. O., \& Spadaro, J. A. (1978). Treatment of orthopaedic infections with electrically generated silver ions. A preliminary report. The Journal of Bone and Joint Surgery, 60(7), 871.

27. Webster, D. A., et al. (1981). Silver anode treatment of chronic osteomyelitis. Clinical Orthopaedics and Related Research, 161, $105-114$.

28. Carr, H. S., Wlodkowski, T. J., \& Rosenkranz, H. S. (1973). Silver sulfadiazine: In vitro antibacterial activity. Antimicrobial Agents and Chemotherapy, 4(5), 585-587.

29. Petrus, E., et al. (2011). A study on the minimum inhibitory concentration and minimum bactericidal concentration of Nano Colloidal Silver on food-borne pathogens. International Food Research Journal, 18(1), 55-66. 